\title{
La iglesia deconstruida y el Jesús alivianado. Inclusión femenina y LGBTQIA+ en tres comunidades cristianas de México
}

\author{
The deconstructed Church and the cool Jesus. Female and LGBTQIA+ inclusion \\ in three Christian communities in Mexico
}

\author{
Carlos S. Ibarra \\ cs.ibarra@live.com \\ El Colegio de la Frontera Norte, México \\ Edson F. Gomes \\ edsonbr.mex@gmail.com \\ Instituto Tecnológico y de Estudios Superiores de \\ Occidente, México
}

Recepción: 28 Febrero 2021

Aprobación: 22 Junio 202

Publicación: 01 Septiembre 2021

Cita sugerida: Ibarra, C. S. y Gomes, E. F. (2021). La iglesia deconstruida y el Jesús alivianado. Inclusión femenina y LGBTQIA + en tres comunidades cristianas de México. Descentrada, 5(2), e145. https://doi.org/10.24215/25457284e145
Resumen: Este artículo analiza los mecanismos de inclusión existentes al interior de tres iglesias postdenominacionales en México que poseen elementos de lo que se denomina iglesia deconstruida. Se argumenta que estas congregaciones promueven condiciones equitativas y anti-discriminatorias para mujeres y también para personas LGBTQIA+ mediante recursos discursivos y materiales que facilitan la detonación de un proceso de deconstrucción religiosa que, a pesar de representar una afrenta a los preceptos cristianos-institucionales tradicionales, logra colaborar con ellos para gozar de legitimidad simbólica dentro de las redes evangélico-pentecostales.

Palabras clave: Cristianismo postdenominacional, Inclusión, Género, Homosexualidad, Derechos LGBTQIA+.

\begin{abstract}
This article analyzes the existing inclusion mechanisms within three postdenominational churches in Mexico that have elements of what is called the deconstructed church. It is argued that these congregations promote equitable and anti-discriminatory conditions for women and also for LGBTQIA + people through discursive and material resources that facilitate the detonation of a process of religious deconstruction that, despite representing an affront to traditional Christian-institutional precepts, manages to collaborate with them to enjoy symbolic legitimacy within the evangelical-Pentecostal networks.
\end{abstract}

Keywords: Postdenominational Christianity, Inclusiveness, Gender, Homosexuality, LGBTQIA+ rights.

\section{INTRODUCCIÓN}

En este artículo se aborda el tema de la inclusión de mujeres y minorías sexuales en congregaciones postdenominacionales que poseen elementos de lo que Marti y Ganiel (2014) denominan iglesia deconstruida. Se parte de las siguientes preguntas de investigación: 1) ¿Cómo se diferencian las iglesias postdenominacionales y la iglesia deconstruida del resto de las iglesias evangélico-pentecostales en términos de los roles femeninos y la inclusión de personas LGBTQIA+?; 2) ¿Cuáles son las diferencias entre lo 
observado en las congregaciones de la iglesia deconstruida y las iglesias para la diversidad sexual y de género (Bárcenas, 2020)?

Para contestar estas preguntas se utilizó un enfoque metodológico basado en la propuesta de la religión vivida, con el propósito de mostrar la existencia de las deconstrucciones propuestas por Marti y Ganiel, observables en los relatos de vida de las personas entrevistadas, en forma de elementos discursivos, así como en los aspectos materiales en la cotidianidad de las personas (Ammerman 2014, 2016; Hall 1997).

Con el fin de contextualizar la propuesta de la iglesia deconstruida, es importante tener en cuenta que, en el mundo anglosajón, las iglesias que no poseen asociación explícita con alguna denominación cristiana establecida suelen clasificarse bajo la categoría de iglesias no-denominacionales. Algo que caracteriza a este tipo de agrupaciones es su relativa independencia operativa, pues no estar sujetas a las directivas de grandes asociaciones religiosas no sólo les permite argumentar ser la iglesia verdadera establecida por Jesucristo, sino también llevar a cabo modificaciones en el culto y alabanza sin depender de un tercero (Miller, 1998). En esa misma línea, las iglesias postdenominacionales también se abstienen de pertenecer a una denominación y tampoco están sujetas a la voluntad de otras organizaciones religiosas, pero poseen un rasgo distintivo que las diferencia de las congregaciones no-denominacionales: rechazan ser detentoras de la verdad absoluta; en cambio, reconocen que toda iglesia posee un fragmento de la verdad, situación que les permite establecer relaciones ecuménicas con más facilidad (Anderson, 2004; Deverell, 2005). En ese sentido, las iglesias deconstruidas que se visitaron durante la fase etnográfica son, en esencia, congregaciones postdenominacionales.

El término iglesia deconstruida hace referencia a comunidades cristianas derivadas del universo evangélico-pentecostal que han surgido en los últimos años y que se caracterizan por haber abandonado ideologías consideradas demasiado moralistas, conservadoras e hipócritas. Una iglesia deconstruida presenta cinco características generales: 1) es anti-institucional, 2) transgrede las fronteras eclesiales y teológicas tradicionales, 3) favorece liderazgos jóvenes, 4) valora la experimentación y la creatividad y 5) crea espacios religiosos neutrales con la intención de evitar parecer, física y espacialmente, templos cristianos tradicionales (Marti y Ganiel 2014). Es preciso señalar que estas iglesias deconstruidas no son lo mismo que las iglesias para la diversidad sexual y de género, pues aunque existe participación de personas con orientaciones sexuales e identidades de género diversas y estas participan activamente en la "reinvención de la Iglesia como institución social" (Bárcenas, 2020, p.11), las estructuras morales del cristianismo no se ven subvertidas en torno a la homosexualidad, como se demostrará con posterioridad.

La relevancia de estas iglesias deconstruidas va en dos sentidos: en primer lugar, pone en evidencia la necesidad de repensar las categorías clasificatorias del campo religioso cristiano mexicano, que ya ha superado la ambigüedad de lo evangélico y lo pentecostal en términos sociológicos y demográficos, sobre todo a la luz de los últimos datos censales; en segundo lugar, las iglesias deconstruidas nos permiten observar cambios drásticos en la religión y en las religiosidades ligados a lo generacional y a lo cultural. Tomando esto en cuenta, vale la pena mencionar que este tipo de congregaciones se encuentran conformadas y lideradas por millennials, los cuales promueven una serie de innovaciones en la forma de concebir, experimentar y representar una religiosidad en armonía con los elementos culturalmente asociados a lo millennial. Un punto de partida para definir qué significa ser millennial son los datos de corte etario; si tomamos esto en cuenta, los millennials serían las personas nacidas entre 1982 y 1996, según lo establece el Pew Research Center. ${ }^{1}$ Esta definición, sin embargo, necesita un contexto particular: la sociedad de los Estados Unidos, con todos los privilegios que ello implica. Así, las características de la población millennial son los siguientes: los millennials son nativos digitales con un marcado sentido de pertenencia a una comunidad global, poseen además un cierto nivel de conciencia sobre asuntos de justicia social, salud y ambiente. Un elemento clave de las y los millennials es que valoran y son sensibles a la diversidad, además de que generalmente denuncian situaciones de injusticia social y cultural; al mismo tiempo que ven favorablemente a las políticas progresivas, valoran la individualidad y poseen una tendencia al emprendedurismo (Fisher 2018; Schewe et al., 2013). Para el caso 
mexicano, dadas las brechas económicas y sociales, la categoría de millennial se reduce a aquellas personas nacidas entre 1982 y 1996 que pertenecen a clases medias y altas y que, además viven en los principales centros urbanos del país, condiciones que les permiten gozar de privilegios en función del tipo de consumo social, cultural y lúdico (De la Garza, López, Guzmán y Atlatenco, 2020; Gómez, Alvarado, Martínez y De León, 2018; Reguillo, 2017).

Las características observadas en las iglesias deconstruidas contrastan además con la manera en que histórica, social y culturalmente se ha tratado a la mujer en contextos religiosos: su invisibilidad dentro del universo judeocristiano suele tomarse como un hecho inalterable, pues salvo algunas excepciones que pueden encontrarse en las escrituras y en algunos relatos derivados del folklor judío -como la historia de Lilith como primera compañera de Adán, expulsada del paraíso por no querer someterse al dominio de este (Wenkart, 1994) - los hilos narrativos que fundamentan la cosmovisión judeocristiana son, estructuralmente, masculinos. A lo largo de los siglos, lecturas específicas de la Biblia -desde el Génesis hasta el Nuevo Testamento- han sido utilizadas para justificar y promover diferentes formas de opresión femenina (Servitje Montull, 2021; Stopler, 2008; Walter y Davie, 1998; Woodhead, 2002). Para el caso de México, el panorama parece desolador: la conjunción de siglos de cultura machista e influencia católica parecen resonar entre sí, produciendo y reproduciendo violencias sistémicas no sólo sobre las mujeres, sino también sobre las minorías sexuales (Stevens y Soler, 1974; Tamayo, 2000; Vaggione, 2009; Vuola, 2006).

Aunque México es un país predominantemente católico, la influencia de Roma ha ido disminuyendo durante las últimas tres décadas: de acuerdo con los datos censales más recientes: sólo un 77,7\% de la población se considera católica; en su lugar, las iglesias evangélico-pentecostales continúan expandiendo su influencia a un ritmo consistente, logrando acaparar un 9,8\% de la población mexicana. Estos números contrastan con los del censo de 2010 , en el que un $82,7 \%$ de la población se consideraba católica y un 7,5\% protestante-evangélica. La cuestión ya ha dejado de ser si México se convertirá en un país protestante. Aunque los datos censales parecieran indicar ese posible futuro, existe una categoría que aumentó de forma considerable respecto al censo de 2010: la gente sin religiónpasó de 5,9\% a 10,6\%, es decir que, hasta 2020, un poco más de 13 millones de mexicanos y mexicanas rechazaron ser parte de un grupo religioso (INEGI, 2020).

Es importante considerar que no todas las personas que se dijeron sin religión carecen de prácticas y creencias trascendentales. Como se verá en este artículo, también es posible que las personas de esta categoría hayan contestado no tener religión y aun así seguir congregándose de manera regular en alguna comunidad cristiana no-denominacional o postdenominacional. Igual de importante es precisar que los datos censales tampoco reflejan necesariamente el estado real de la diversidad en las identidades religiosas en el país, sino que son una forma cuantitativa de visualizar la institucionalización de las creencias, como bien lo señalan Algranti, Mosqueira y Setton (2019) para el caso argentino.

La historia de la teoría de la secularización es útil para entender cómo es posible que gente sin religión sea, efectivamente, parte de iglesias postdenominacionales y no-denominacionales. La teoría de la secularización fue útil para explicar las transformaciones religiosas en Europa durante su periodo de industrialización y hasta la primera mitad del siglo XX: en esos momentos, las sociedades occidentales se encontraban inmersas en el discurso de la modernidad y del progreso lineal, sobre todo a la luz de los avances tecnológicos, sociales y científicos, de tal forma que era natural predecir el fin de la religión (Berger, 2014). Entre las señales de la inminente secularización del mundo se encontraban, por ejemplo, la comercialización y mercantilización de celebraciones originalmente ligadas a lo religioso, como la navidad (Belk, 1987; Bock, 1972), o el declive de prácticas ligadas a una religiosidad tradicional, como las oraciones y los rezos antes de consumir alimentos, de dormir o de leer fragmentos de Biblia en el seno del hogar (Bossard y Boll, 1976).

Una vez fracturada la ilusión de progreso, las ciencias sociales comenzaron a observar cambios y contradicciones que no podían explicarse ya mediante el enfoque de la secularización: si bien la religión no iba a desaparecer, las condiciones bajo las que la gente creía y practicaba su religiosidad comenzaron a reconfigurarse. Entre los elementos que se pueden identificar como cambios importantes en las religiosidades 
cristianas occidentales a partir de la década de 1970 se encuentran los siguientes: el incremento en asistencia a congregaciones no-tradicionales y un decremento en los números de feligreses que asistían a iglesias tradicionales. Este fenómeno también se vio reflejado en el abandono de vestimentas tradicionales, la sustitución de la música tradicional por música contemporánea y la participación de personas no ordenadas en los cultos de adoración. Tres ejemplos concretos respecto a esto pueden ser hallados en la modernización de las alabanzas en las iglesias evangélicas y pentecostales, sobre todo en lo que respecta al uso de instrumentos que antes eran considerados profanos, como la batería y las guitarras eléctricas (Ramírez, 2015), o en la utilización de la radio, la televisión y las redes sociales para transmitir rituales sagrados en espacios considerados profanos (O 'Guinn y Belk, 1989).

Ante estos cambios, la operatividad del concepto de religión propuesto por Hervieu-Léger resulta útil para deslindar definiciones sustantivistas y funcionalistas de este fenómeno. Para esta autora, es imposible generar una definición última de religión. En vez de eso, propone que "una religión es un dispositivo ideológico, práctico y simbólico, a través del cual se constituye, mantiene, desarrolla y controla la conciencia (individual y colectiva) de la pertenencia a un linaje creyente particular" (Hervieu-Léger, 2005, p. 137). Es decir que una forma de entender el fenómeno religioso en la actualidad es observando la relación que existe entre la tradición imperante y las formas de creer de las personas; de este choque es que surgirán procesos de recomposición religiosa.

Comprender por qué alguien que asiste a congregaciones postdenominacionales y/o no-denominacionales podría contestar que no tiene religión, implica bajar las recomposiciones religiosas que propone HervieuLéger al terreno del sentido práctico. Una de las formas más estudiadas en México en lo referente a este fenómeno es la de la religión popular y la religión a la carta (De la Torre, 2012), pero para adquirir un mayor nivel de precisión en términos de agencia individual, este artículo aborda el fenómeno del rol de las mujeres y las minorías sexuales en las iglesias deconstruidas desde el marco de la religión vivida, con el objetivo de describir y analizar las estrategias de inclusión que se emplean no sólo a nivel organizacional, sino también en el ámbito de la vida cotidiana, a pesar de las restricciones que suelen existir para las mujeres y minorías sexuales al interior del cristianismo.

\section{Metodología}

Las entrevistas que nutren a este artículo surgen de varios periodos de trabajo de campo en el sur de los Estados Unidos y en México entre los años 2016 y 2020, específicamente en las ciudades de Tijuana, Ensenada, Guadalajara, Los Ángeles y San Diego. Estas incursiones etnográficas fueron parte de dos tesis de posgrado: una de maestría y otra de doctorado (Gomes, 2020a; Ibarra, 2019). Si bien la temática de este artículo no era el objetivo principal de ambas investigaciones, parte de los hallazgos giraron en torno a la promoción y puesta en práctica de condiciones equitativas y anti-discriminatorias para mujeres y personas LGBTQIA+ en este tipo de iglesias, mediante recursos discursivos y/o materiales que facilitaban la detonación de procesos de deconstrucción religiosa. Las personas entrevistadas fueron abordadas en diferentes contextos, ya fuese en la misma iglesia, en sus casas y en cafeterías de las diferentes ciudades exploradas. Las entrevistas realizadas en México provienen de tres iglesias: Ancla (Tijuana), Horizonte (Ensenada) y Más Vida (Guadalajara). En los Estados Unidos, las entrevistas se llevaron a cabo en Ancla (San Diego) y MOSAIC (Los Ángeles).

Para la selección de participantes en las investigaciones originales, el único criterio que se tomó en cuenta fue el de elegir a miembros regulares de estas iglesias, considerando que, para dichos estudios, el trabajo de campo era mayormente exploratorio ante la carencia de investigaciones previas respecto a este tipo de congregaciones. El uso de una aproximación metodológica como la religión vivida nos permite centrarnos en las prácticas y experiencias cotidianas de las personas que se asumen como seguidores o practicantes de alguna religión, filosofía religiosa o creencias en lo sobrenatural y trascendental (Ammerman, 2016; Hall 1997). ¿Qué hacen en su día a día? ¿Qué creen realmente? ¿Cómo lo implementan en su cotidiano? ¿Cómo se visten? 
¿Qué comen? ¿Cómo se divierten? ¿Por qué lo hacen de esa manera? Dado el nivel de individualización en la experiencia religiosa que se vive al interior de las iglesias deconstruidas, utilizar este tipo de metodología nos permite observar, en la práctica y en el discurso, la forma en la que las personas apropian y viven las enseñanzas que circulan al interior de estas comunidades. Tomando esto en cuenta y utilizando un guion de entrevista, los relatos de cada participante fueron encaminados hacia elementos materiales, discursivos y corporales de su vivencia religiosa, con el fin de tener puntos de referencia generales para el análisis posterior. Esta estrategia permitió que, durante las conversaciones, todas las personas hablaran sobre su interpretación individual del evangelio y la puesta en práctica en su día a día de estas creencias. Como se podrá observar en la sección con los fragmentos de las diez entrevistas realizadas, el eje principal de la deconstrucción religiosa pasa por una renegociación de la barrera entre lo sagrado y lo profano.

\section{CONSTRUCCIÓN SIMbóliCA DE GÉNERo Y LIDERAZgo PROTESTANTE TRADICIONAL}

Dado que la iglesia deconstruida deriva del universo evangélico-pentecostal y, a su vez, este se origina dentro del protestantismo, es importante comprender cómo se ha abordado el rol tradicional de la mujer y de las minorías sexuales en el cristianismo protestante latinoamericano. Es inevitable partir del hecho de que el cristianismo es inherentemente patriarcal y de que las mujeres han sido remitidas a un papel secundario. Para el caso de las minorías sexuales, las escrituras se han utilizado durante siglos para justificar la persecución y la criminalización de la homosexualidad, según lo indica la Ley Mosaica (Faggioni, 2013). Históricamente, el rol femenino dentro del protestantismo se ha caracterizado por una cierta ambivalencia (Bauberot, 1994) que se desprende de las ideas sobre la educación que debía recibir la mujer en los tiempos de la Reforma Protestante y del puritanismo y, por otro lado, de la interpretación bíblica sobre las formas de organización social patriarcales, en las cuales el hombre goza de privilegios en detrimento de la mujer. En este mismo sentido, Amestoy refiere que en la primera mitad del siglo XIX la preocupación por la instrucción femenina colocó a los países angloamericanos y protestantes a la cabeza. No obstante, en la segunda mitad del mismo siglo, "a partir de un concepto patriarcal de los roles, se levantó un vallado contra el voto femenino que abría paso para el acceso al ministerio y la ordenación pastoral” (2012, p. 55). Así, el rol tradicionalmente asociado a la mujer cristiana es el de ser una ayuda idónea para el hombre, por lo que esta suele poseer un papel secundario, a la sombra del protagonismo del varón, de manera análoga con la forma en la que los roles femeninos han estado culturalmente arraigados en la sociedad: la mujer como ama de casa, la mujer como madre, la mujer como trabajadora doméstica, la mujer como única responsable del cuidado de los hijos, etcétera.

Los reavivamientos pentecostales del siglo XIX y XX en los Estados Unidos significaron un cambio importante: las mujeres no sólo comenzaron a tomar roles de liderazgo en sus comunidades religiosas, sino que la posibilidad de su participación se expandió en términos eclesiásticos (Ramírez, 2015). Sin embargo, estos nuevos roles no representaron liderazgos auténticos sobre grupos mixtos de feligreses, sino una manera de exacerbar más la brecha sexogenérica al interior de las congregaciones, pues únicamente podían presidir grupos compuestos por mujeres. Independientemente de ello y de manera sutil, la posibilidad de liderazgos femeninos comenzó a propagarse, sobre todo con la difusión de las ideas sobre democracia que tuvieron lugar a partir del siglo XVIII (Amestoy, 2012). A pesar de estos avances, el status quo de la mujer no cambió de manera drástica entre las denominaciones protestantes y evangélico-pentecostales hasta la llegada de la segunda mitad del siglo XX, de la mano con los procesos migratorios que fomentaron la aparición de lideresas comunitarias que, fungiendo como cabezas de familia ante la ausencia de sus parejas, comenzaron a romper paradigmas en términos de los roles sociales y culturales permitidos para las mujeres (Ramírez, 2015).

Para entender los nuevos roles de la mujer en el cristianismo protestante latinoamericano actual es importante describir dos tendencias generales opuestas: los cristianismos conservadores y los cristianismos emergentes. Aunque en países como México, el grupo de cristianos conservadores está constituido 
principalmente por los protestantismos históricos, los grupos evangélico-pentecostales y, de manera más reciente, los grupos carismáticos/neopentecostales, en otros países de América Latina, como sucede con el caso de Argentina, los protestantismos históricos han integrado la bandera de los derechos sexuales y reproductivos a su agenda teológica (Tarducci, 2018). Más allá de estas particularidades, los cristianismos conservadores suelen estar asociados al crecimiento político de la derecha y consolidan una corriente marcada por un sentimiento de nostalgia de tiempos en los cuales las normas socioculturales se encontraban ligadas de manera cercana con los deberes cívico-religiosos judeocristianos (Almeida, 2017). El grupo de los cristianismos emergentes está constituido en su mayoría por grupos postdenominacionales y nodenominacionales, que han hecho concesiones y acercamientos al mundo secular en términos de alabanzas y experiencias religiosas mejor adaptadas a las tendencias igualitarias de las sociedades occidentales modernas. Los cristianismos emergentes también contemplan a las iglesias para la diversidad sexual y de género que han sido observadas en países como Brasil, México y Estados Unidos (Bárcenas, 2020; Machado y Delvalhas Piccolo, 2011; Sumerau, 2017). Así, este tipo de congregaciones suelen representar una tendencia más alineada a una vena política progresiva, reflejada en su preocupación por resolver el dilema de las desigualdades y las injusticias sociales, tratando de hacer énfasis en los pasajes y enseñanzas bíblicas que hablan sobre la gracia y el perdón divino, en vez del fuego, la ira y la condena. Aunque los cristianismos emergentes son grupos que viven en conflicto con las aparentes contradicciones que surgen de sus intentos por emparejar las escrituras con el mundo profano, existen congregaciones que han logrado generar comunidades innovadoras gracias a su capacidad para resolver en mayor o menor medida estos conflictos, tocando temas considerados tabú para el cristianismo en general, como la igualdad de género, el derecho al aborto, el tema de la sexualidad y la homosexualidad, entre otros (Gomes, 2020a; Ibarra, 2019).

\section{LA iglesia deconstruida en MÉXico}

A pesar de su marcada identidad católica, México ha sido un país laico desde 1857 y no fue hasta la declaración de la Ley de Asociaciones Religiosas y Culto Público de 1992 que se comenzó a visibilizar la diversidad religiosa del país, al fundarse la Oficina de Asuntos Religiosos, encargada de registrar a toda asociación y grupo religioso en territorio mexicano. En 2020 ya se habían registrado más de 7000 congregaciones de diversas denominaciones, siendo cerca de la mitad de ellas de origen católico (De la Torre, Gutiérrez y Hernández, 2020). De cierta manera, la promulgación de esta ley fue un parteaguas para el campo religioso mexicano en términos de visibilidad de la diversidad religiosa en el país: en diez años, la adscripción a iglesias protestantes y, particularmente, a las iglesias evangélico-pentecostales, se incrementó por millones, aunque es importante aclarar que el establecimiento de esta oficina no implica una correlación directa con el devenir de la diversidad religiosa en México, la cual está sujeta a procesos históricos y sociales complejos. Así, para entender de dónde surge la idea de lo postdenominacional y de la iglesia deconstruida, debemos remitirnos, de manera general, a la trayectoria de este tipo de congregaciones.

El universo evangélico-pentecostal no es un bloque homogéneo. Su diversificación comenzó desde el momento en que hizo su arribo en tierras mexicanas y, sin embargo, luego de varios reavivamientos a lo largo de dos siglos (Bastian, 2013; Cox, 2009; Creech, 1996; Dow, 2005; Ramírez, 2015; Robeck, 2017), los grupos evangélico-pentecostales lograron posicionarse de manera importante entre las clases bajas, la población rural y la población indígena, principalmente por su habilidad para proveer redes de apoyo y sentido de pertenencia entre estos grupos (Garrard-Burnett y Stoll, 1993). El factor migración, por otro lado, disparó la presencia evangélico-pentecostal en el país, gracias al establecimiento de comunidades translocales y transfronterizas entre aquellas personas que emigraron a los Estados Unidos y sus comunidades de origen. No es coincidencia que los estados del norte y los estados del sur del país posean la concentración más grande de cristianos evangélico-pentecostales (Hernández y O 'Connor, 2013; Odgers y Ruiz, 2009; Ramírez, 2003; Stephen, 2007). Además de ello, la frontera entre México y Estados Unidos es el corredor más importante 
para el tráfico de drogas en la región, por lo que el problema de las adicciones ha contribuido también al crecimiento de los grupos evangélico-pentecostales, particularmente a través de los centros de rehabilitación administrados por ellos (Odgers y Olivas, 2018).

El elemento transfronterizo es fundamental para el surgimiento de la iglesia deconstruida en México, pues los primeros grupos del cristianismo emergente que implementaron esta perspectiva surgieron en el sur de California dada la composición multiétnica e híper-diversificada de dicho estado. California no sólo ha sido el receptor de la mayor cantidad de flujos migratorios provenientes de México y Latinoamérica, es también uno de los estados norteamericanos con una clara tendencia favorable hacia políticas progresistas en lo social y lo cultural, de tal manera que tres agrupaciones religiosas, aprovechando estos elementos contextuales, significaron una especie de primordio para la noción de una iglesia deconstruida: el Jesus Movement, la Vineyard Christian Fellowship y Calvary Chapel; grupos que, a pesar de su naturaleza conservadora, comenzaron a hacer concesiones con el fin de resultar atractivos para las juventudes californianas que sentían afinidad con los movimientos hippies y sus nociones de libertad desde los años setenta (Ibarra, 2019; Shibley, 1996).

Lo transfronterizo es fundamental para comprender el establecimiento de la iglesia deconstruida en México. Una persona transfronteriza se refiere a quien, de manera regular y casi cotidiana, cruza la frontera México-Estados Unidos por motivos de trabajo, compras o recreación, ya sea con papeles de residencia o ciudadanía norteamericana o bien, con visas de turista. Dado lo frecuente de su inmersión en las culturas nacionales de ambos países, lo transfronterizo implica una hibridez sociocultural que genera manifestaciones materiales y simbólicas particulares (Alegría, 2015). El desarrollo histórico de las congregaciones evangélicopentecostales en el sur de California, en conjunción con las dinámicas transfronterizas, así como las comunidades translocales establecidas entre México y Estados Unidos, dieron lugar a la creación de redes de soporte evangélico-pentecostales entre ambos países, las cuales permiten instancias de colaboraciones oficiales y no-oficiales entre diferentes congregaciones evangélicas, pentecostales, neopentecostales, nodenominacionales y postdenominacionales. Estas redes brindan legitimidad, dentro del universo cristiano evangélico, a todas aquellas iglesias que se mantienen en los márgenes de lo tradicionalmente aceptado (Ibarra, 2021).

En la actualidad, una de las congregaciones que más ha marcado a las iglesias postdenominacionales en la región es MOSAIC en la ciudad de Los Ángeles: aunque originalmente fue fundada como una iglesia bautista en los años cuarenta, su localización geográfica y las dinámicas culturales y sociales propias del sur de California, sumadas al trabajo hecho por los movimientos arriba mencionados, hicieron que esta se volviera cada vez más diversa en términos étnicos, sociales y culturales (Marti, 2009). En la década de 2010 entró en un periodo de renovación audiovisual, aprovechando el auge de las plataformas digitales y la intersección entre religión y cultura pop. Tres iglesias abordadas en este artículo se han visto influenciadas por MOSAIC. Dos se encuentran en el noroeste de México: Ancla, en las ciudades de Tijuana y San Diego; Horizonte en la ciudad de Ensenada y Más Vida en la ciudad de Guadalajara (Ibarra, 2019; Gomes, 2020a).

Es importante aclarar que la noción de iglesia deconstruida no necesariamente se encuentra presente en todas las congregaciones postdenominacionales, aunque uno o varios elementos de ella pueden estar presentes incluso en agrupaciones no-denominacionales y en algunas otras iglesias evangélico-pentecostales con un componente considerable de población joven, particularmente de clase media y alta (Rocha, 2017). Aquellas congregaciones que implementan la totalidad de los elementos que Marti y Ganiel (2014) enunciaron pueden concebirse como iglesia deconstruida. Las ideas sobre lo divino y sobre Dios que las personas asistentes a estas iglesias suelen tener, surgen del mismo lugar desde donde se originan otras teologías cristianas: la Biblia. La diferencia entre las iglesias deconstruidas y otras congregaciones postdenominacionales, nodenominacionales o evangélico-pentecostales es tajante, ya que mientras los segundos entienden que es un deber cristiano el separarse del mundo, los primeros conciben que son parte del mundo y su deber como personas cristianas es, en parte, el de llevar la cultura del reino a esos espacios abandonados por el cristianismo 
(Aguiar, 2020). Otro elemento que distingue a este tipo de congregaciones es que la interpretación de los textos sagrados no es literal, pues se adapta simbólicamente a los tiempos y a la cultura que se esté encargando de llevar a cabo dicha interpretación.

Materialmente, las congregaciones abordadas en este artículo tienen un dominio sobre las plataformas digitales y tienen equipos de medios compuestos por miembros que son estudiantes o profesionales del diseño gráfico y las artes visuales, desarrollo de software, fotografía e ingeniería en audio, entre otras disciplinas, por lo que el tipo de producciones que surgen al interior de este tipo de congregaciones va más allá de una simple modernización de la alabanza o la predicación, como sucede en muchas iglesias evangélico-pentecostales y neopentecostales de clase media y alta. Es a través de estos recursos que resuenan con los usos y tipos de consumo cultural de toda una generación, lo cual incrementa la atracción para personas que no son tradicionalmente religiosas (Ibarra, 2019). En ese mismo sentido, dos elementos fundamentales inmateriales que han echado raíz en las iglesias deconstruidas tienen que ver con la no discriminación de cualquier tipo y con la sensibilización hacia la diferencia (Howe y Strauss, 2000). En los fragmentos de entrevistas que se presentan en este artículo hay cuatro elementos determinantes en la cotidianidad de las personas en términos de materialidad y discurso: las creencias fundamentales, las creencias secundarias, la madurez en la fe y el no ser piedra de tropiezo.

Las creencias fundamentales o primarias son aquellas creencias no-negociables, independientemente del tipo de congregación cristiana, y se pueden resumir en el siguiente pasaje bíblico: "pues Dios amó tanto al mundo que dio a su único Hijo, para que todo el que crea en él no se pierda, sino que tenga vida eterna" (Juan 3:16, Nueva Traducción Viviente). Es decir que las creencias fundamentales consisten en la idea central a toda congregación cristiana: que el sacrificio de Jesús limpió el pecado de toda la humanidad y le salvó por gracia divina; nada que el humano haga podrá cambiar ese hecho (Kimball, 2003). Este es un punto que genera polémica entre el resto de las congregaciones del universo evangélico-pentecostal pues, durante las investigaciones de campo realizadas en ese tipo de iglesias entre 2016 y 2020 en México y los Estados Unidos, las personas que asistían y eran parte de esas iglesias deconstruidas a menudo eran acusadas de tener una comprensión superficial y conveniente de las escrituras.

Las creencias secundarias son las cuestiones de forma que cada iglesia tiene en su interpretación de la palabra de Dios (Ibarra, 2019): así como los pentecostales creen en los dones del espíritu, o los testigos de Jehová rechazan transfusiones sanguíneas, las iglesias deconstruidas piensan, entre otras cosas que:

Los cristianos hemos pasado mucho tiempo temiéndole al mundo, una creación divina; lo tenemos abandonado y hemos permitido que el mal se apropie de muchos espacios; nuestro deber es reapropiarnos de la creación, dejar de temerle a los espacios que nos han dicho que no son cristianos (Entrevista a Juan, pastor).

Este posicionamiento es un indicador del proceso renegociación de la división entre lo sagrado y lo profano. Si para Durkheim el elemento distintivo de una religión era la separación entre lo sagrado y lo profano (Durkheim, 2008), en el caso de la iglesia deconstruida la separación sigue existiendo, pero se ha desplazado para abarcar elementos que otras congregaciones siguen prohibiendo; esto explica por qué al interior de este tipo de congregaciones sigue existiendo una concepción del pecado similar a la que está presente en todo el cristianismo, pero en simbiosis con la idea de que toda la creación no sólo es de origen divino, sino hecha para el disfrute humano. En ese sentido, el límite definitorio entre el plano de lo sagrado y el plano de lo profano se redefine en función de la intencionalidad que las personas tienen respecto a sus acciones.

El tercer elemento que circula al interior de las iglesias deconstruidas tiene que ver con una madurez en la fe; con este término se hace referencia a lo siguiente: "Mientras más conoces las escrituras y la gracia de Dios, más libre te sientes para llevar a cabo diferentes cosas que pueden ser muy criticadas por personas legalistas" (Juan; pastor, entrevista). Es decir, una persona inmersa en una iglesia deconstruida será capaz de llevar a cabo un mayor número de acciones y actividades "profanas" en función de qué tan seguro se encuentre de su relación personal con Dios. El nivel de madurez en la fe les permite tener una postura liberal en torno 
a temas relevantes para su generación. Esta libertad dada por la madurez en la fe se encuentra socialmente regulada mediante el concepto de no ser piedra de tropiezo, término con el que se describe el cuidado que las personas maduras en la fe deben tener para no afectar a terceros que puedan estar teniendo una lucha o un problema con elementos del mundo secular:

El cristiano inmaduro suele creerse fuerte, y acusa de débiles e inmaduros a quienes beben alcohol o disfrutan del mundo... de verdad creo que ese grado de libertad viene desde la iglesia, una persona que sabe disfrutar de su libertad sin abusar de ella (Entrevista a Benjamín, pastor).

La totalidad de las personas entrevistadas en las iglesias en las que se llevó a cabo trabajo de campo (Horizonte en Ensenada, Ancla en Tijuana y San Diego, MOSAIC en Los Ángeles y Más Vida en Guadalajara) relataron una visión de Jesús mucho más ligera que la versión predominante en iglesias tradicionales:

El humano le pone tantos rituales y tantos rollos a algo que para Jesús simplemente era como, not a big deal; imagino a Jesús diciendo algo así como 'quiero que disfruten entre ustedes y que me adoren y que declaren vida y que sí también, como dicen en la Biblia, que vayan a todo el mundo y declaren el evangelio, declaren esta nueva noticia, pero disfrutando la vida' (Entrevista a María, asistente).

Dentro de esta visión de un Jesús que ve las cosas de una forma más alivianada, la cuestión de frecuentar lugares como bares, antros y otros espacios tradicionalmente considerados profanos no parece ser un problema:

Una vez hicimos una posada y llevaron botellas de vino tinto, entonces hubo una persona que, pues se incómodo ¿sabes? y bueno yo dije 'pues es vino tinto, pues Jesús compartió el vino' y hubo una vez que escuché una prédica que decía 'bueno, ¿por qué crees que invitaron a Jesús? O sea, por qué crees que ir con los novios o el anfitrión principal, pues porque él era el alma de la fiesta, sabían que él lo iba a poder hacer' (Entrevista a Victoria, asistente).

La operatividad de la madurez en la fe se observa cuando las personas se ven confrontadas con situaciones ambiguas en términos tradicionalmente cristianos:

Por mi convicción yo sé que mi relación con Dios está bien, si yo voy a un lugar con mis amigos y yo salgo, y si yo no me condeno por eso, pues está bien, pero si tú te condenas por eso entonces a lo mejor ahí estás mal, ¿sabes? Porque te sientes culpable porque yo creo que la intención de Dios no es que tú te sientas culpable ni mucho menos que tú te condenes por algo que tú puedas hacer. Si tú te condenas entonces ya va a ser una cosa que tú ya vas a tener que trabajar contigo mismo y con Dios (Entrevista a Victoria, asistente).

Respecto a no ser piedra de tropiezo, Gabriela menciona lo siguiente:

Quizá a lo mejor va a haber una persona nueva o que viene de una iglesia super legalista o una persona que a lo mejor viene de... quizá fue alcohólico y viene aquí para mejorarse y de repente sale con los chicos de la alabanza y los chicos de la alabanza, 'vamos a dónde están unas cheves ¿o qué?’ Y dices 'no manches ¿qué onda?' entonces bueno, por compasión y prudencia, a lo mejor no me va a tocar beber en frente de esa persona para quizá cuidar su corazón (Entrevista a Gabriela, asistente).

Así, las iglesias deconstruidas son un quiebre con el universo evangélico-pentecostal, pues además de que no utilizan elementos simbólicos ni litúrgicos tradicionales promueven un acercamiento con el mundo secular, por lo que son capaces de generar estéticas de persuasión que se encuentran en armonía con las expectativas cognitivas y culturales de los jóvenes involucrados. Por estéticas de persuasión se hace referencia a la propuesta de Meyer (2010) respecto a la concepción del éxito del cristianismo pentecostalizado en tanto que se presenta como un medio que permite somatizar, en términos sensoriales, la experiencia de la salvación y el espíritu santo. Para el caso de las congregaciones analizadas, las estéticas de la persuasión están presentes en lo material, en lo simbólico y en lo cotidiano. Un ejemplo de ello es la ya mencionada ausencia de elementos tradicionales: no existe un púlpito, sino una plataforma minimalista que se asemeja a los escenarios dispuestos para espectáculos de stand up comedy; la vestimenta y la apariencia de los pastores, líderes y asistentes se encuentra fuertemente influenciada por la moda hipster urbana; la música, los cánticos y la performatividad 
de la banda que lidera las alabanzas y del público que escucha se asemeja a un show de música pop-indie de la escena artística secular.

El proceso más relevante para este artículo, sin embargo, es el de la deconstrucción religiosa, que ocurre cuando los preceptos teológicos del cristianismo son readaptados a través de la creencia en una madurez en la fe, la idea de no ser piedra de tropiezo, y la diferenciación entre creencias fundamentales y creencias secundarias. Estos tres elementos detonan una serie de innovaciones y cambios de lo que, teológicamente hablando, implica ser cristiano: de pronto ya no se tiene una urgencia por ser santo, ni por evangelizar al resto del mundo para provocar la segunda venida de Jesucristo; tampoco se le teme al mundo ni al pecado. Las consecuencias de esta deconstrucción tienen como consecuencia la creación de comunidades inclusivas, abiertas a la diferencia y a la diversidad en todos sus aspectos, sin tener que posicionarse explícitamente en términos políticos sobre un tema en particular.

\section{IGLESIA DECONSTRUIDA E INCLUSIÓN}

Dos temas álgidos para cualquier congregación cristiana suelen ser la cuestión de la homosexualidad y la cuestión de la inclusión y libertad femenina. Respecto a lo primero, las iglesias deconstruidas consideran que la homosexualidad es un pecado, pero disminuyen su importancia al contextualizar que todos los humanos son pecadores por naturaleza y que Dios ha dado perdón de estos a través de su sacrificio. Esta aproximación es sencilla pero estratégica, pues facilita la convivencia teológica entre las partes más y menos progresistas de estas comunidades:

...hablando específicamente de la comunidad gay, la Biblia es un libro antiguo, lo más reciente es de hace casi dos mil años, y es nuestra base de inspiración. Los cristianos tenemos que estar dispuestos a decir: 'nuestra mentalidad se forja por los escritos que nosotros creemos que son inspirados por Dios'. Eso significa que van a haber cosas, que va a haber un choque entre lo que nosotros creemos y lo que la cultura predica... tengo amigos jóvenes que ves su Facebook y está lleno de comentarios cien por ciento homofóbicos, y como cristiano eso me da un chorro de pena, porque eso está mal; porque antes de ser homosexuales, son humanos. Y debe haber una barra de humanidad que nos una sin importar que tan diferentes sean nuestras creencias. Hay cosas que no sé si es palabra en español, pero que dehumanizan a la comunidad gay, que yo creo que no se deberían decir de ningún ser humano... cualquier persona homosexual es bienvenida en Horizonte, no tienen que cambiar lo que creen para venir, tenemos convenciones muy distintas a lo que puedan creer, y obviamente es nuestro deseo que lleguen a la verdad a través de la Biblia, pero no tenemos un filtro que dice que únicamente puedes venir si eres heterosexual... tenemos personas sirviendo en la iglesia que son abiertamente gay y no tenemos ningún problema con eso; quisiera que llegaran a lo que yo creo es una convicción bíblica de su sexualidad, pero si no la tienen, de todas formas son hijos de Dios... yo no les diría '¡Arrepiéntete!', les diría: 'eres amado por Dios, y eres amado por esta comunidad'... bíblicamente esto que te voy a decir es muy polémico, pero es lo que es: la homosexualidad es un pecado, pero ¿sabes qué más es un pecado? Tener sexo antes del matrimonio, estar en rebeldía, mentir, herir a otras personas, ser soberbio, avaro, y muchas otras actitudes que todos hacemos... y sin embargo la gracia de Dios nos salva y nos perdona... el pecado es rebeldía, y no he conocido a una persona homosexual que sea homosexual sólo por estar en rebeldía... (Entrevista a Juan, pastor).

Respecto al papel de la mujer, se debe mencionar que uno de los libros más polémicos de la Biblia es el Levítico, no sólo por haber sido utilizado históricamente para oprimir y justificar la invisibilización de la mujer y otras minorías sexuales, sino también para dictar qué elementos habrán de ser estigmatizados por la comunidad cristiana. En las congregaciones estudiadas, sin embargo, hay una lectura particular de dicho libro:

¿Puede tatuarse un cristiano? es muy polémica porque hay un versículo en Levítico que dice que los levitas no se deben de marcar en memoria de los muertos, que no deben de marcar su cuerpo y muchas traducciones dicen 'tatuar su cuerpo' , entonces, levítico dice claramente que no nos podemos tatuar, entonces ¿estoy yendo en contra de la ley, tatuándome? Yo creo una cosa: que Levítico y esa zona de Levítico está hablando acerca de los funcionarios del templo de los levitas, no la definición que tenemos hoy en México de levita, de alguien que dirige la alabanza, sino los levitas, los que están haciendo las funciones del templo, que ellos no deben de tatuarse, ¿por qué? Porque era en ese entonces, y realmente aún en nuestra cultura hasta hace poco, una señal de estarte asociando con dioses paganos, entonces ¿cómo vas a asociarte con dioses paganos y llegar a la iglesia a hacer las cosas necesarias para que las personas puedan tener una relación con Dios? Pero, yo creo en lo 
personal que eso es para los levitas, entonces, si no eres levita, no creo que aplique a ti, pero, pero, ya sé que hay personas que automáticamente dicen: ‘ipero está en la Biblia!’. Ok, si recorres unos cuantos versículos antes, en ese pasaje, dice que no puedes usar dos diferentes tipos de tela, entonces si tú estás usando $25 \%$ algodón y $75 \%$ poliéster, entonces estás en pecado como si te hubieras puesto un tatuaje (Entrevista a Juan, pastor).

La reinterpretación del Levítico en función de los tiempos actuales es un giro que no todas las congregaciones cristianas se atreven a hacer abiertamente, particularmente desde sus líderes y pastores, sobre todo cuando hay una postura muy específica en contra de marcar un cuerpo. Al preguntarle a un pastor de Más Vida sobre la participación de jóvenes abiertamente homosexuales en la iglesia, ofreció una explicación similar a la de Juan:

Sí, nuestras creencias son muy claras, la Biblia lo dice, pero creemos también lo que Dios puede hacer, es decir que nosotros no descartamos o limitamos a una persona por sus creencias era lo que te decía, le damos la bienvenida a toda persona como vengan, de dónde vengan; que hicieron, que no hicieron, pero creemos en Dios, que él puede transformar sus vidas, es algo que no obligamos simplemente dejamos que Dios lo haga. Eso es parte de nuestro funcionamiento como iglesia, no forzamos nunca el que alguien crea algo, sino que Dios plante en su corazón ese sentido o ese camino que Dios tiene para esa persona (Entrevista a Antonio, pastor).

Con esto, Antonio deja claro su posicionamiento y el posicionamiento de la institución: aunque no dejan de considerar a la homosexualidad como un pecado, su postura hacia las personas homosexuales es de no segregación, no discriminación y, sobre todo, la no recriminación ni la búsqueda directa o compulsiva para “curar" a una persona de la comunidad LGBTQIA+. Si bien la persuasión para la transformación de una cualidad no deseada podría ser interpretada como discriminación, este tipo de iglesias no imponen castigos o tratos diferenciados para quienes no cambian, pues se respetan los procesos espirituales individuales. El no condenar ni auto condenarse es parte fundamental de la convivencia en este tipo de congregaciones. Así, una de las asistentes a Más Vida comentó:

No estamos cerrados en el sentido de que todos son bienvenidos. Algo que me encanta que nuestro pastor dice mucho es que amamos a Dios y amamos a las personas. Entonces no me toca a mí juzgar como eso, cerrarle las puertas a alguien, porque así como Dios ama esas personas, Dios me ama a mí, o sea Dios me ama con todo y mis fracasos y mi pasado y todo, pero Dios no quiere dejarme en esa misma situación. Entonces, si amamos a las personas, no tenemos problema con que personas de ese tipo vengan (Entrevista a María, asistente).

Como se ha descrito anteriormente, por más que no dejen sus raíces de entendimiento teológico reformado sobre los principios bíblicos, el cambio que se da en las iglesias deconstruidas va sobre las formas, particularmente sobre la manera en la que tratan a las minorías en función del enfoque que le dan a la gracia divina y el perdón otorgado por Jesús (Gomes, 2020a). Esta misma persona indicó que en Más Vida las personas buscan vivir lo que ellos resumen en su eslogan: "ven como eres":

No estamos jamás en contra de quien comete el pecado, llámale homosexualidad, llámale robo, lo que sea, cualquier pecado. Estamos en contra del pecado, no del pecador y lo que queremos es eso que cada persona tenga un encuentro con Jesús, que cada persona pueda llegar a encontrar su verdadera identidad en Jesús, sin necesidad de ser regañados de ser atacados a bibliazos casi casi... creemos que el proceso espiritual también de cada persona es súper diferente, puede que haya una persona que desde la primera vez que vino tuvo un cambio radicalmente su vida, pero puede que haya otras que no, que tal vez necesitan más tiempo (Entrevista a María, asistente).

En pocas palabras, los puntos que se observan sobre la homosexualidad en este tipo de iglesias tienen que ver con: 1) normalizar el ser pecador, no como un problema, sino como una condición inherente a todas las personas; 2) a pesar de ello, no dejan de entender como pecado concepciones de identidades sexogenéricas distintas a las bíblicas; 3 ) dan énfasis a la gracia salvadora de Jesús, a través de la cual ningún pecado es lo suficientemente grande para no ser perdonado; 4) el trato que se le da al público LGBTQIA+ es de igualdad, sin que su identidad sexogenérica sea un problema.

En ese mismo sentido, la igualdad de condición de la mujer se reinterpreta de la siguiente manera: 
Génesis 1:26-27 nos dice que los días de la creación se acercan a su fin... la humanidad, el hombre y la mujer reciben dominio sobre la creación; ambos fueron creados a la imagen de Dios, no sólo el hombre, sino también la mujer, y se les asigna la misma tarea, no se dice 'los varones gobernarán la tierra y las mujeres estarán bajo el mando o subordinadas a los hombres', pero seguro dirán ‘ah, pero ella fue creada para ser su ayudante ¿no implica eso sumisión?’; bueno, esa es una forma de verlo. Que Adán necesitase ayuda no era una señal de autoridad sobre la mujer, la palabra 'ayudante' o 'compañero' se utiliza en otras partes de la Biblia para describir a Dios mismo... Dios es llamado 'nuestra ayuda', nuestro 'escudo ', nuestra 'fuerza' cuando se asocia con la palabra 'ayuda'. Es la misma palabra que en hebreo se usa para describir a Eva... Pablo al decir que las mujeres deben cubrirse la cabeza también dice que profetizar y orar deben hacerse de cierto modo... mi interpretación del pasaje es que Pablo está utilizando muchos elementos culturales de su tiempo y de su contexto cultural... en ese mismo pasaje les recuerda a sus lectores que todo es para Dios, y que todo hombre que ha existido después del primero lo ha hecho gracias a las mujeres... nos recuerda que nos necesitamos los unos a los otros... cubrirse la cabeza no es un símbolo de inferioridad, es la marca del derecho y la autoridad para participar... tienen autoridad como mujeres... cuando los hombres restringen a las mujeres y oprimen su voz, están restringiendo la gloria y el honor que Dios puso sobre ellas, están interfiriendo con el orden que dicen querer mantener (Entrevista a Roberto, pastor).

Se puede apreciar que hay un proceso de reinterpretación bíblica para justificar el hecho de que hombres y mujeres tienen las mismas posibilidades de autoridad al interior de la iglesia. No es extraño que en iglesias como Ancla, Horizonte, Más Vida y MOSAIC existan no sólo liderazgos femeninos, sino también pastoras y mujeres en posiciones de poder en términos administrativos: la persona que funge como tesorera y contadora en Ancla es mujer y la diseñadora jefe de Horizonte es mujer, por mencionar dos ejemplos. Mientras otras iglesias debaten el papel de las mujeres al interior de ellas, para las iglesias deconstruidas la distinción entre hombre o mujer es irrelevante (Ibarra, 2019). Una de las líderes entrevistadas comentó lo siguiente respecto a ser mujer en una iglesia deconstruida:

Para mí, lo más importante que he aprendido es que Jesús entendió nuestra condición humana y nos liberó; no pienso que hacer cosas del mundo te haga menos deseable ante sus ojos... una de las cosas que me gustan de esta comunidad es que es, probablemente, el safe space más increíble que he tenido la oportunidad de conocer; creo que la postura en contra de la discriminación realmente se practica al interior, no por nada es una iglesia inclusiva... nunca en los casi cinco años que llevo aquí, he sentido que mi condición de mujer haya tenido peso en las posibilidades que están a mi alcance... si eso no es feminismo entonces no sé qué más será (Entrevista a Aubrey, líder).

\section{El caso de Beatriz, en Tijuana, cuenta una experiencia similar a la de Aubrey:}

El momento de ruptura con mi congregación anterior vino cuando me convertí en mamá; como fue por fuera del matrimonio, sentí mucho rechazo... quizá no directamente, pero sí podía sentir las miradas y los chismes... la gente se fija tanto en esos detalles y pierden de vista lo importante... en ese aspecto prefiero Ancla. Me gusta pensar que la mayoría sabemos que todos tenemos cola que nos pisen y que ultimadamente, lo que nos mantiene unidos es que somos hijos de Dios y que ya estamos salvos; no sé si seamos más maduros en nuestra fe que otros hermanos en Cristo, de mi parte sí puedo decir que he intentado ir más allá y cuestionar realmente la forma en las que abordo mi fe y la forma en la que la vivo en este mundo... no pienso que el cristianismo sea incompatible con la modernidad, con la posmodernidad, el cristianismo es amor y tratar de comprender las diferencias entre todos... sé que no todos están de acuerdo en todos los aspectos pero el saber distinguir entre creencias primarias y secundarias es muy importante para desarrollar esa madurez y no caer en anacronismos como la satanización del sexo, del alcohol o de las drogas, ojo que no digo que esté a favor de esto ni que lo haga, pero la gente hace cosas por muchas razones y no debemos juzgar... si tengo más oportunidades acá que en otros lados, simple y llanamente sí, creo que aquí no hay nada de diferencia en cuanto a tus aspiraciones dentro de la organización de la iglesia por el simple hecho de ser mujer, me gusta mucho la reinterpretación de ese pasaje donde Pablo dice que la mujer debe servir al hombre, y que se ha utilizado por otras organizaciones para justificar que la mujer no pueda ser líder o aspirar a puestos de poder en la iglesia... para mí no hay ningún tipo de mecanismo que permita una invisibilidad de la mujer en Ancla y esa es otra cosa que me gusta mucho de esta comunidad (Entrevista a Beatriz, líder).

El caso de Renata, líder en Horizonte, es un ejemplo de las oportunidades que reciben las mujeres al interior de este tipo de iglesias:

Yo tomé el curso de liderazgo y quiero convertirme en una líder fuerte para demostrarle a las mujeres que ven con escepticismo a la religión que es posible ser una mujer empoderada, con voz de mando, al interior de una iglesia... los tiempos han cambiado 
y la discriminación no es algo que pueda tener lugar en un lugar en donde se enseña el amor al prójimo (Entrevista a Renata, líder).

Casos como este son ejemplo de la intención que hay para generar liderazgos jóvenes sin restricciones sexo-genéricas. Si bien es cierto que la inclusividad observada en este tipo de congregaciones puede parecer insignificante si se compara con las medidas y transformaciones que otras organizaciones de la sociedad civil han implementado en los últimos años, o con las observadas en iglesias para la diversidad sexual y de género, el hecho de que comunidades religiosas usualmente asociadas a conservadurismos político-sociales estén en proceso de readaptación es un fenómeno interesante que debe ser tomado en cuenta dadas las implicaciones que este tipo de congregaciones tienen para las formas de creer a futuro.

\section{Conclusiones}

Retomando las preguntas de partida de este artículo, podemos decir que los resultados encontrados en torno al tema de la inclusión femenina y LGBTQIA+ en las congregaciones analizadas son los siguientes: las iglesias postdenominacionales promueven una apertura ecuménica mayor que cualquier otro tipo de agrupación religiosa; reconocer que no detentan la verdad absoluta y que todas las congregaciones cristianas tienen el potencial para salvar a la humanidad las coloca en una posición de ventaja en términos de negociación entre las esferas conservadoras y liberales dentro del cristianismo. Como se argumentó al inicio de este artículo, una manifestación particular de lo postdenominacional es la iglesia deconstruida propuesta por Marti y Ganiel. La noción de una iglesia deconstruida y la generación de cristianismos emergentes dentro de las manifestaciones postdenominacionales en México permiten abordar la práctica religiosa desde un contexto contemporáneo que atiende la conjunción de elementos seculares relevantes, como pueden ser manifestaciones de la cultura pop, el uso cotidiano de redes sociales y tecnología, o temas de relevancia social, como los derechos humanos y la diversidad, los cuales son factores clave para comprender la transición que puede ocurrir entre grupos evangélicos y pentecostales hacia formas innovadoras de concebir la salvación, generalmente encabezadas por millennials inmersos en un mundo digital, tecnológico y extremadamente influenciado por la comunicación y la ya mencionada cultura pop (Gomes, 2020a; Ibarra, 2019; Marti y Ganiel, 2014).

Algo que caracteriza a las iglesias deconstruidas es la búsqueda por reapropiarse del mundo profano bajo la lógica de que los cristianos se han apartado del mundo, el cual es considerado una creación divina, por lo que han dejado espacio para que el mal reine en él (Ibarra, 2019). Esta forma de concebir el deber cristiano también puede ser observada en la misión que tienen las iglesias postdenominacionales por liderar una conquista, no sólo de espacios, sino de la propia cultura "profana" para el reino de Dios, así manifestando la cultura del reino en la tierra (Aguiar, 2020). En términos generales, las congregaciones de la iglesia deconstruida no pretenden conquistar cultura y ambientes seculares con la intención de transformarlos, sino para el goce de estos por parte de sus miembros, evitando generar sentimientos de culpa, de vergüenza o bien procesos de auto-victimización. En ese sentido, desplazan estratégicamente la barrera tradicional entre lo sagrado y lo profano mediante una serie de negociaciones personales en función de la distinción entre creencias fundamentales, creencias secundarias y el nivel de madurez en la fe.

Otro elemento importante para comprender las reconfiguraciones generadas por la iglesia deconstruida tiene que ver con la concepción de pertenecer a una comunidad de personas afines, o practicar un estilo de vida, más que ser miembros de una institución religiosa en sí. Para algunas de las personas entrevistadas, el ser cristiano no es motivo suficiente para asegurar que pertenecen a una religión en sí:

Pues, como te digo, Dios no está peleado con eso (ir a bares y consumir alcohol). ¿Sabes? Hay veces que creo que religiosamos tanto, por eso te digo yo no creo tener, seguir, una religión sino una relación con Dios (Entrevista a Victoria, asistente).

Como se pudo ver en la sección anterior, uno de los pilares que refuerzan el funcionamiento de la comunidad y de las creencias al interior de este tipo de congregaciones tiene que ver con la idea de crear 
un estilo de vida afín a las expectativas generacionales de los millennials en términos sociales, políticos, culturales e identitarios, evitando así conflictos y generación de culpa con la manera en la que el mundo secular se conforma actualmente. No olvidemos que el cristianismo, en general, se caracteriza por sentimientos, acciones y pensamientos que centran su atención más en sentimientos de culpa que de goce y salvación (Gauthier, 2018).

Tampoco puedes religiosar [sic] todo, no somos de palo y tampoco somos extraterrestres. Dios sabe que la vamos a regar, por algo en la Biblia dice en Salmos que sus misericordias son nuevas cada mañana, otra versión dice que 'su perdón es nuevo cada mañana’ ¿Por qué crees que te perdona todos los días a cada mañana? Porque sabe que la vas a regar, porque sabe que no somos de palo, porque sabe que su amor es tan infinito que dice 'yo te perdono, no hay bronca, empezamos de cero otra vez, dale' (Entrevista a Victoria, asistente).

Considerando esto, debe tenerse en cuenta que el crecimiento millonario de las personas sin religión en el censo de población de México en 2020 puede deberse también, en parte, a este tipo de iglesias deconstruidas, las cuales rechazan la idea de crear instituciones tradicionalmente u oficialmente religiosas. Con este contexto es que surgen los procesos deconstructivos ejemplificados por las nociones de creencias fundamentales, creencias secundarias, madurez en la fe y no ser piedra de tropiezo, que a su vez permiten tener aproximaciones más progresivas al tema de la inclusión de público LGBTQIA + y femenino, sin tener que centrar sus esfuerzos en este tipo de comunidades de manera exclusiva, así como a la generación de una estructura organizacional de corte más horizontal que favorece la inclusión, legitimación, visibilidad y participación de mujeres dentro de posiciones de poder al interior de estas comunidades.

Entender cómo se diferencian las congregaciones de la iglesia deconstruida de las iglesias para la diversidad sexual y de género implica posarse sobre la relevancia y la naturaleza del primer tipo de comunidades en materia de inclusión: las "deconstrucciones" observadas en estas congregaciones no poseen el mismo origen epistemológico ni ontológico que los procesos de deconstrucción en comunidades seculares, como sucede en el ámbito académico. Asimismo, la iglesia deconstruida fundamenta la inclusión femenina y LGBTQIA+ desde un punto de vista teológico judeocristiano. Esto implica que las identidades sexo-genéricas difícilmente perderán el estigma simbólico de pecado que históricamente han cargado dentro del cristianismo. Tampoco existe un cuestionamiento oficial sobre subvertir la concepción binaria del género. La estrategia es, desde luego, extender la categoría de pecado hacia toda actividad humana, de tal forma que ser homosexual tiene la misma carga negativa que otras características y actividades de la vida cotidiana que son consideradas como pecado, por lo menos desde un punto de vista estrictamente bíblico. Estas características hacen que las congregaciones estudiadas entren en la categoría de "iglesia inclusiva” que proponen autoras como Bárcenas, Machado y Delvalhas Piccolo; en tanto que no son iglesias para la diversidad sexual y de género, pues no están integradas ni estructuradas exclusivamente o específicamente para personas LGBTQIA+ (Bárcenas 2020; Machado y Delvalhas Piccolo, 2011).

En conclusión, los mecanismos de renegociación entre lo sagrado y lo profano que se observan en las congregaciones de la iglesia deconstruida son estrategias particulares que, si bien corren el riesgo de minimizar o invisibilizar procesos y conflictos identitarios en términos sexo-genéricos, también contribuyen a la sensibilización del universo evangélico-pentecostal, no-denominacional y postdenominacional en cuestiones de diversidad. Las redes de apoyo evangélico-pentecostales en las que participan estas iglesias contribuyen a la diseminación de este tipo de posturas de corte progresista, como se demuestra en los productos que han resultado de las etnografías utilizadas para este artículo (Gomes, 2020b; Ibarra, 2021).

\section{ReFERENCIAS}

Aguiar, T. P. (2020). A "cultura" para o Reino: materialidades e sentidos da adoração em uma juventude evangélica em Porto Alegre (Tesis de maestría). Universidade Federal do Rio Grande do Sul. Porto Alegre, Brasil. 
Alegría, T. (2015). Metrópolis transfronteriza: revisión de la hipótesis yevidencias de Tijuana, México y San Diego, Estados Unidos. Tijuana: El Colegio de la Frontera Norte.

Algranti, J., Mosqueira, M. y Setton, D. (2019). Pensar sin Iglesias: el hecho institucional como problema de estudio. La institución como proceso. En J. Algranti, M. Mosqueira, y D. Setton (Ed.). Configuraciones de lo religioso en las sociedades contemporáneas (pp. 29-58). Buenos Aires: Biblos.

Almeida, R. D. (2017). A onda quebrada-evangélicos e conservadorismo. Cadernos Pagu, 50. https://doi.org/10.159 $0 / 18094449201700500001$

Amestoy, N. (2012). Las mujeres en el protestantismo rioplatense 1870-1930. Franciscanum. Revista de las Ciencias del Espiritu, 54(157), 51-81. Recuperado de https://dialnet.unirioja.es/descarga/articulo/4560989.pdf

Ammerman, N. (2014). Finding Religion in Everyday Life. Sociology of Religión, 75(2), 189-207. Recuperado de http s://academic.oup.com/socrel/article-abstract/75/2/189/1652239

Ammerman, N. (2016). Lived religion as an emerging field: an assessment of its contours and frontiers. Nordic Journal of Religion and Society, 29(2), 83-99. Recuperado de https://pdfs.semanticscholar.org/e1f7/3c0f4fe6107ca827 b6caleee93d4cff66256.pdf

Anderson, A. (2004). An Introduction to Pentecostalism: Global Charismatic Christianity. Oxford: Oxford University Press.

Bauberot, J. (1994). La mujer protestante. En G. Duby et al. (Ed.). Historia de las mujeres (Tomo VII, pp. 219-233). Madrid: Taurus.

Bárcenas, K. (2020). Bajo un mismo cielo. Las Iglesias para la diversidad sexual y de género en un campo religioso conservador. México: Universidad Nacional Autónoma de México-Instituto de Investigaciones Sociales.

Bastian, J.P. (2013). Protestantismos y modernidad latinoamericana: Historia de unas minorias religiosas activas en América Latina. México: Fondo de Cultura Económica, México.

Belk, R. (1987). A Child's Christmas in America: Santa Claus as Deity, Consumption as Religion. Journal of American Culture, 10(1), 87-100. Recuperado de https://onlinelibrary.wiley.com/doi/abs/10.1111/j.1542-734X.1987.1 001_87.x

Berger, P. (2014). The Many Altars of Modernity: Toward a Paradigm for Religion in a Pluralist Age. Boston: De Gruyter.

Bock, E. (1972). The Transformation of Religious Symbols: A Case Study of St. Nicholas. Social Compass, 19(4), 537-548. Recuperado de https://journals.sagepub.com/doi/abs/10.1177/003776867201900403

Bossard, J. y Boll, E. (1976.) Ritual in family living: a contemporary study. Connecticut: Greenwood Press.

Cox, H. (2009). Fire from Heaven: The Rise of Pentecostal Spirituality and the Reshaping of Religion in the 21st Century. Cambridge: Da Capo Press.

Creech, J. (1996). Visions of glory: The place of the Azusa Street revival in Pentecostal history. Church History, 65(3), 405-424. https://doi.org/10.2307/3169938

De la Garza, M., López J., Guzmán, E. y Atlatenco, Q. (2020). Validation of a measuring scale of the factors for the employability of millennials. Gadjah Mada International Journal of Business, 22(2),178-198. Recuperado de ht tps://search.informit.org/doi/10.3316/informit.426862757180556.

De la Torre, R. (2012). La religiosidad popular como "entre-medio" entre la religión institucional y la espiritualidad individualizada. Civitas, 12(3), 506-521. Recuperado de https://www.journals.openedition.org/pontourbe/58

De la Torre, R., Gutiérrez, C., y Hernández, A. (2020). Religious reconfiguration in Mexico: Beliefs and Practices National Survey, 2016. Social Compass, 67(3), 349-371. https://doi.org/10.1177/0037768620922122

Deverell, G. (2005). Uniting in Worship? Proposals towards a Liturgical Ecumenics. Uniting Church Studies, 11(1), 21-36. Recuperado de http://cp.unitingchurch.org.au/uniting_in_worship.pdf

Dow, J.W. (2005). The expansion of Protestantism in Mexico: An anthropological view. Anthropological Quarterly, 78(4), 825-851. Recuperado de http://www.jstor.org/stable/4150963

Durkheim, E. (2008). Las formas elementales de la vida religiosa (Trad. A. Martínez). Madrid: Alianza Editorial. 
Faggioni, M. (2013). La comunidad cristiana y la homosexualidad. Moralia, 36(137), 73-110. Recuperado de http://web.b.ebscohost.com/ehost/pdfviewer/pdfviewer?vid=4\&sid=668d105e-d179-4569-8f38-a55f6ba $5722 \mathrm{~d} \% 40$ sessionmgr 101

Fisher, P. (2018). A Political outlier: the distinct politics of the millennial generation. Society, 55(1), 35-40. Recuperado de https://doi.org/10.1007/s12115-017-0209-7

Garrard-Burnett, V.y Stoll, D. (1993). Rethinking Protestantism in Latin America. Filadelfia: Temple University Press.

Gauthier, F. (2018). "Our Play Pleases the Man, the Spirits of the Desert, and Whatever". Enjoying religion at Burning Man. En F. Jespers et al (Ed.). Enjoying Religion. Pleasure and Fun in Established and New Religious Movements (pp. 103-126). Lanham: Lexington Books.

Gomes, E. F. (2020a). Cuando la fe se vuelve marca: la comunicación organizacional y el cristianismo emergente en el caso "Más Vida Guadalajara"(Tesis de maestría). Instituto Tecnológico y de Estudios Superiores de Occidente. Tlaquepaque, México.

Gomes, E. F. (2020b). ADN Más Vida: la identidad de una iglesia postdenominacional mexicana en crecimiento. Ciencias Sociales Y Religión/Ciências Sociais E Religiäo, 22, e020035. https://doi.org/10.20396/csr.v22i00.138 07

Gómez, D., Alvarado, L., Martínez, M. y Díaz de León, C. (2018). La brecha digital. Una revisión conceptual y aportaciones metodológicas para su estudio en México. Entreciencias: Diálogos en la Sociedad del Conocimiento, 6(16): 49-64. https://doi.org/10.22201/enesl.20078064e.2018.16.62611

Hall, D. (1997). Lived Religion in America: Toward a History of Practice. Princeton: Princeton University Press.

Hernández, A. y O 'Connor, M. (2013). Migración y conversión religiosa entre los mixtecos de Oaxaca. Alteridades, 23(45), 9-23. Recuperado de http://www.scielo.org.mx/scielo.php?script=sci_abstract\&pid=S0188-70172013 $000100002 \& \operatorname{lng}=\mathrm{es} \& \mathrm{nrm}=\mathrm{iso}$

Hervieu-Léger, D. (2005). La Religión, Hilo de Memoria. Barcelona: Herder Editorial.

Howe, N. y Strauss, W. (2000) Millennials Rising: The Next Generation. Nueva York: Vintage Books.

Ibarra, C.S. (2019). Cristianismo Postdenominacional, Movimientos Emergentes y Deconstrucción Religiosa en el Norte de México (Tesis de doctorado). El Colegio de la Frontera Norte. Tijuana, México.

Ibarra, C.S. (2021). Beards, tattoos, and cool kids. Lived Religion and Postdenominational Congregations in Northwestern Mexico. International Journal of Latin American Religions. https://doi.org/10.1007/s41603-02 $1-00133-7$

Kimball, D. (2003). The Emerging Church: Vintage Christianity for New Generations. Grand Rapids: Zondervan.

Marti, G. (2009). A mosaic of believers: diversity and innovation in a multiethnic church. Bloomington: Indiana University Press.

Marti, G. y Ganiel, G. (2014). The deconstructed Church: understanding emerging Christianity. Oxford: Oxford University Press.

Machado, M. y Delvalhas Piccolo, F. (2011). Religióes e Homosexualidades. Rio de Janeiro: FGV Editora.

Meyer, B. (2010). Aesthetics of persuasion: global Christianity and Pentecostalism's sensational forms. South Atlantic Quarterly, 109(4), 741-763. https://doi.org/10.1215/00382876-2010-015

México. Instituto Nacional de Estadística y Geografía. (2020). Censo de Población y Vivienda 2020. Recuperado de h ttps://www.inegi.org.mx/programas/ccpv/2020/

Miller, D. (1998). Postdenominational Christianity in the Twenty-first century. The Annals of the American Academy of Political and Social Science, 558(1), 196-210. https://doi.org/10.1177/0002716298558001015

Odgers, O. y Olivas, O. (2018). ¿Dejar las drogas con ayuda de Dios? Experiencias de internamiento en centros de rehabilitación fronterizos. Tijuana: El Colegio de la Frontera Norte.

Odgers, O. y Ruiz, J. (2009). Migración y Creencias: Pensar las Religiones en Tiempo de Movilidad. Tijuana: El Colegio de la Frontera Norte.

O 'Guinn, T. y Belk, E. (1989). Heaven on Earth: Consumption at Heritage Village, USA. Journal of Consumer Research, 16(2), 227-238. https://doi.org/10.1086/209211 
Ramírez, D. (2003). Migrating Faiths or Transgenic Danger? Pentecostal Growth in Oaxacalifornia. Conferencia presentada en el Latin American Studies Association Meeting, Dallas, Texas, Estados Unidos. Recuperado de https://www.academia.edu/10826169/Migrating_Faiths_or_Transgenic_Danger_Pentecostal_Growth_in _Oaxacalifornia

Ramírez, D. (2015). Migrating Faith: Pentecostalism in the United States and Mexico in the Twentieth Century. Chapel Hill: University of North Carolina Press.

Reguillo, R. (2017). Los jóvenes en México. México: Fondo de Cultura Económica.

Robeck, C.M. (2017). The Azusa Street mission and revival: The birth of the global Pentecostal movement. Nashville: Thomas Nelson.

Rocha, C. (2017). "The Come to Brazil Effect": Young Brazilians ' Fascination with Hillsong.En T. Riches et al. (Ed.) The Hillsong Movement Examined: You Call Me Out Upon the Waters (pp. 125-141). Londres: Palgrave Macmillan.

Schewe, C., Debevec, K., Madden, T., Diamond, W., Parment, A., y Murphy, A. (2013). "If you 've seen one, you 've seen them all!" are young Millennials the same worldwide? Journal of International Consumer Marketing, 25(1), 3-15. https://doi.org/10.1080/08961530.2013.751791

Servitje Montull, M. (2021). Genealogía crítica de la violencia: Hacia la liberación del espacio politico-religioso del cuerpo de las mujeres. México: Universidad Iberoamericana.

Shibley, M. (1996). Resurgent Evangelicalism in the United States: Mapping Cultural Change Since 1970. Columbia: University of South Carolina Press.

Stephen, L. (2007). Transborder Lives: Indigenous Oaxacans in Mexico, California and Oregon. Durham: Duke University Press.

Stevens, E. y Soler, M. (1974). El marianismo: la otra cara del machismo en América Latina. Diálogos: Artes, Letras, Ciencias Humanas, 10(1), 17-24. Recuperado de https://www.jstor.org/stable/27933189

Stopler, G. (2008). A rank usurpation of power. The role of patriarchal religion and culture in the subordination of women. Duke Journal of Gender Law \& Policy, 15(1), 365-398. Recuperado de https://scholarship.law.duke.e $\mathrm{du} / \mathrm{djglp} / \mathrm{vol} 15 /$ iss $1 / 5$

Sumerau, J. (2017). "Some of Us Are Good, God-Fearing Folks". Justifying Religious Participation in an LGBT Christian Church. Journal of Contemporary Ethnography, 46(1), 3-29. https://doi.org/10.1177/08912416145 59142

Tamayo, S. (2000). La ciudadanía civil en el México de la transición: mujeres, derechos humanos y religión. Revista Mexicana de Sociología, 62(1), 61-97. https://doi.org/10.2307/3541179

Tarducci, M. (2018). Escenas claves de la lucha por el derecho al aborto en Argentina. Salud colectiva, 14, 425-432. h ttps://doi.org/10.18294/sc.2018.2036

Vaggione, J. (agosto, 2009). Sexualidad, religión y politica en América Latina. Conferencia presentada en Diálogos Regionales. Río de Janeiro, Brasil. Recuperado de http://sxpolitics.org/ptbr/wp-content/uploads/2009/10/sex ualidad-religion-y-politica-en-america-latina-juan-vaggione.pdf

Vuola, E. (2006). ¿Gravemente perjudicial para su salud? Religión, feminismo y sexualidad en América Latina y el Caribe. Pasos, 127, 15-26. Recuperado de http://biblioteca.clacso.edu.ar/Costa_Rica/dei/20120712012320/g ravemente.pdf

Walter T. y Davie, G. (1998). The religiosity of women in the modern West. British Journal of Sociology, 49(4), 640-660. https://doi.org/10.2307/591293

Wenkart, H. (1994). Feminist Revaluation of the Mythical Triad, Lilith, Adam, Eve: A Contribution to Role Model Theory. Philosophy in the Contemporary World, 1(4), 40-44. https://doi.org/10.5840/pcw19941426

Woodhead, L. (2002). Religions in the modern world: Traditions and Transformations. Hove: Psychology Press

\section{Notas}

1 https://www.pewresearch.org/ 